\title{
TrkA Inhibitor VMD-928
}

National Cancer Institute

\section{Source}

National Cancer Institute. TrkA Inhibitor VMD-928. NCI Thesaurus. Code C153116.

An orally bioavailable, selective inhibitor of tropomyosin receptor kinase A (TrkA; neurotrophic tyrosine receptor kinase (NTRK) type 1; NTRK1; TRK1-transforming tyrosine kinase protein), with potential antineoplastic activity. Upon oral administration, VMD-928 specifically targets and binds to TrkA, inhibits neurotrophin-TrkA interaction and prevents TrkA activation. This prevents the activation of downstream signaling pathways and inhibits cell growth in tumors that overexpress TrkA. Uncontrolled TrkA signaling plays an important role in tumor cell growth, survival, invasion and treatment resistance. 